\title{
Expectativas y trayectorias educativas postsecundarias de jóvenes de territorios rurales en Chile. Una mirada desde el desarrollo humano
}

Jorge Castillo-Peña

\section{RESUMEN}

$\mathrm{El}$ artículo analiza la relación entre educación y territorio respecto a las diferencias en las aspiraciones y trayectorias educativas universitarias de los adolescentes de centros educativos urbanos y de zonas rurales, y las estrategias que diseñan estos centros para resolver las carencias de oportunidades en sus territorios. Para ello se toma como marco teórico referencial el enfoque de desarrollo humano y, metodológicamente, el análisis de trayectorias educativas. Los resultados muestran que la expectativa por completar una carrera en la universidad ha aumentado entre los estudiantes rurales, lo que reduce la brecha de expectativas en relación con aquellos de grandes conglomerados urbanos. No obstante, el tránsito hacia ella sigue presentando desigualdades. La migración emerge como la principal estrategia en los territorios rurales, la que es percibida como necesaria, aunque implique un alto costo económico y emocional.

Palabras clave: educación superior, educación rural, trayectoria escolar, Chile. 
Expectativas e trajetórias educativas pós-secundarias de jovens de territórios rurais no Chile. Um olhar a partir do desenvolvimento humano

\title{
RESUMO
}

O artigo analisa a relação entre educação e território com relação às diferenças nas aspirações e trajetórias educativas universitárias dos adolescentes de centros educativos urbanos e de zonas rurais, e as estratégias que desenham estes centros para resolver as carências de oportunidades em seus territórios. Para isso se toma como marco teórico referencial o enfoque de desenvolvimento humano e, metodologicamente, a análise de trajetórias educativas. Os resultados mostram que a expectativa de completar uma carreira na universidade tem aumentado entre os estudantes rurais, o que reduz a brecha de expectativas em relação com aqueles de grandes aglomerados urbanos. Contudo, o caminho até ela continua apresentando desigualdades. A migração emerge como a principal estratégia nos territórios rurais, que é percebida como necessária, ainda que implique um alto custo econômico e emocional.

Palavras chave: educação superior, educação rural, trajetória escolar, Chile.

\section{Expectations and post-secondary educational trajectories of young people from rural areas in Chile. A human development perspective}

\begin{abstract}
This article analyzes the relationship between education and territory with regard to the differences in the aspirations and university educational trajectories of adolescents from urban and rural educational centers, and the strategies designed by these centers to solve the lack of opportunities in their environment. For this purpose, the human development approach is used as a theoretical frame of reference and, methodologically, the analysis of educational trajectories. The results show that the expectation of completing a university degree has increased among rural students, which reduces the expectations gap in relation to those of students from large urban conglomerates. However, the transition to university keeps being discriminatory. Migration emerges as the main strategy in rural territories, which is perceived as necessary, although it implies a high economic and emotional cost.
\end{abstract}

Keywords: higher education, rural education, school trajectory, Chile. 


\section{Introducción}

En los últimos 25 años, el acceso a la educación superior $^{1}$ en Chile ha crecido significativamente a causa de la cobertura alcanzada en enseñanza secundaria, la diversificación en la oferta de programas e instituciones de educación superior (ES) y una ampliación de las alternativas de financiamiento, como becas y créditos (Brunner, 2015).

En 2016, según datos del Consejo Nacional de Educación (GNED), 1161222 alumnos estudiaban en el sistema de educación superior, casi el doble de la matrícula total registrada en 2005 (CNED, 2016). En esta misma línea, el indicador de cobertura en educación superior del Informe de Desarrollo Social, señala que en el primer quintil de ingresos $(20 \%$ más pobre), los jóvenes que accedían a la universidad pasaron de un 4\% en 1990 a un $27.4 \%$ en 2013. En el segundo quintil, pasó de $6.3 \%$ en 1990 a un $30.5 \%$ durante 2013, mientras que en el quintil más rico, pasó de 33.1\% en 1990 a 57.7\% en 2013 (Ministerio de Desarrollo Social, 2015b). Esto implica un cambio en las expectativas y aspiraciones de los jóvenes, con una mayor pretensión a seguir estudiando.

Esta expansión se observa también entre jóvenes que habitan distintos tipos de territorios. En la tabla
1 se muestra el bajo porcentaje de jóvenes de zonas rurales que asistían a alguna institución de educación superior en 2003. En 2015, la tasa se triplicó, alcanzando el $27 \%$ y acortando la brecha de acceso que existe entre el territorio urbano y rural.

Este mayor acceso a la ES ha influido en las expectativas de las personas. La ES hoy aparece en las representaciones de los chilenos como uno de los ejes más importantes para el desarrollo individual y social (PNUD, 2012, 2017; Puga et al., 2017), lo que se expresa de manera más aguda en el segmento juvenil: adolescentes y jóvenes ven la educación como una herramienta que les permitirá desplegar sus proyectos de vida, lograr sus metas, ser valorados socialmente y lograr autonomía e independencia (INJUV, 2017; Castillo, 2013). Se sustenta también en las aspiraciones salariales, ya que la población con mayor nivel educativo obtiene mayores beneficios. Así se expresa en los resultados de la Encuesta Casen (MDS, 2015b), donde una persona que ha terminado la enseñanza secundaria tiene un promedio de ingreso de $\$ 320285^{2}$ mensuales de su ocupación principal, mientras que aquellos que tienen estudios superiores logran duplicar dicho monto, alcanzando un ingreso de $\$ 657468^{3}$ promedio (Ministerio de Desarrollo Social, 2015a).

Tabla 1. Porcentaje de la población de 18 a 24 años que asiste a una institución de educación superior (IES)

\begin{tabular}{|c|c|c|c|}
\hline Año & Urbano & Rural & $\begin{array}{c}\text { Brecha } \\
\text { (puntos porcentuales)* }\end{array}$ \\
\hline 2003 & $28 \%$ & $8 \%$ & -20 \\
\hline 2006 & $29 \%$ & $11 \%$ & -18 \\
\hline 2009 & $31 \%$ & $15 \%$ & -16 \\
\hline 2011 & $37 \%$ & $19 \%$ & -18 \\
\hline 2013 & $39 \%$ & $22 \%$ & -17 \\
\hline 2015 & $39 \%$ & $27 \%$ & -12 \\
\hline
\end{tabular}

Fuente: elaboración propia a partir de datos del Ministerio de Desarrollo Social, Encuesta Casen 2003-2015.

${ }^{*}$ Diferencia estadísticamente significativa al $5 \%$ en todos los años analizados.

\footnotetext{
${ }^{1}$ Educación superior incluye educación técnico profesional y universitaria.

${ }^{2}$ Equivalente a unos 391 USD, aproximadamente.

${ }^{3}$ Equivalente a unos 802 USD, aproximadamente.
} 
Quienes mayormente han cambiado sus pretensiones a futuro son jóvenes pertenecientes a segmentos de niveles socioeconómicos más bajos o de sectores rurales que antes no optaban a estudios superiores, principalmente por su capital económico y cultural, y que hoy le demandan al sistema mayor inclusión y oportunidades que les permitan pensar en proyectos de vida más ambiciosos (UDP, 2016a).

La educación tiene un rol clave en el proceso de desarrollo de las personas al favorecer el despliegue de sus potencialidades. En la escuela, niños y adolescentes desarrollan saberes, actitudes y habilidades que los ayudarán a definir y llevar a cabo sus metas y aspiraciones para la vida. Los propios jóvenes son quienes exigen de la escuela más apoyo para aprender a definir y llevar a cabo sus proyectos (PNUD-UNICEF, 2014), así como también más oportunidades que ayuden a mitigar las desigualdades de origen (Concha, 2013).

Este artículo analiza la relación entre educación y territorio, dando luces sobre las siguientes interrogantes: ¿existen diferencias en las aspiraciones educacionales universitarias de los adolescentes según el territorio donde habitan? ¿Existen diferencias en las trayectorias educativas entre aquellos que habitan en zonas rurales y aquellos provenientes de centros urbanos? ¿Cómo planean resolver los jóvenes rurales y sus familias las carencias de oportunidades que tienen sus territorios para construir sus proyectos de vida?

Primero, se examina cómo el ingreso a la educación superior forma parte de las aspiraciones y de los proyectos de vida de los adolescentes de distintos tipos de territorio. Luego, se comparan las trayectorias de una cohorte de estudiantes que habitan territorios de diferente escala para conocer si tienen la misma probabilidad de alcanzar determinados niveles educativos. Finalmente, se muestra cómo a partir de la desigual distribución de oportunidades territoriales emergen discursivamente entre los adolescentes, proyectos de vida en los que la migración se vuelve la principal estrategia para concretar las aspiraciones de una mayor educación.

\section{Marco conceptual}

El marco teórico de esta investigación se enmarca en el enfoque de desarrollo humano, más conocido como el enfoque de las capacidades ("capabilities approach") que enlaza la calidad de vida y el bienestar, con la libertad. Desde esta perspectiva, el desarrollo humano consiste en el proceso de aumentar las oportunidades de las personas, es decir, eliminar algunos tipos de ausencia de libertad que dejan a los individuos pocas opciones y escasas oportunidades para llevar el tipo de vida que tiene razones para valorar. En ese sentido, el "desarrollo" de una sociedad debería propender a superar estos problemas, y ello sólo se puede lograr aumentando la libertad de los individuos, lo cual incrementa su agencia, mejorando la capacidad para dirigir sus propias vidas y para influir en el mundo (Sen, 1999).

Para Sen, la capacidad de una persona hace referencia a las combinaciones alternativas de funcionamientos que les resulta factible de alcanzar. En ese sentido, la capacidad viene a ser la libertad sustantiva de alcanzar combinaciones alternativas de funcionamientos. Es decir, una capacidad para constituirse requiere de una oportunidad existente en el entorno social, y la libertad de la persona de elegir adquirirla o interiorizarla como recurso. Muchas veces esa libertad no existe, y las decisiones se definen a través de "preferencias adaptativas"; cuando la sociedad ha puesto ciertas cosas fuera del alcance de algunas personas, éstas normalmente aprenden a no querer esas cosas. Otro factor a considerar en este enfoque es que el no optar por un funcionamiento o por una interiorización puede no ser efectivamente una elección consciente (Nussbaum, 2012).

La noción de capacidades que surge de los trabajos de Amartya Sen, ha sido profundizado también por Martha Nussbaum, quien al preguntarse “Qué es capaz de hacer y de ser cada persona?, interroga no sólo por el estado de bienestar de una persona, sino también por las "oportunidades disponibles" que existen para cada ser humano, otorgando una 
relevancia central al rol de las sociedades (de los Estados y las políticas públicas) en la dotación de dichas oportunidades. De esta forma, la calidad de vida que una sociedad ofrece a las personas depende de las capacidades posibles, es decir, de las oportunidades que ofrece (Nussbaum, 2012).

Promocionar estas capacidades permite dotar a las personas de "libertades reales" para que puedan realizar aquello que efectivamente desean y no estar necesariamente determinados por el medio en el que se desenvuelven. Cuando la persona es capaz de integrar ese recurso en la creación de su biografía estamos hablando de que posee o ha adquirido una capacidad. Se entiende, entonces, que contar con capacidades relevantes refuerza la agencia de los sujetos, los vuelve más autónomos y proactivos para perseguir su bienestar (Sen, 1999; Nussbaum, 2012; PNUD, 2012).

La otra parte de una capacidad es su "funcionamiento", que es la realización activa de una o más capacidades. Son "seres" y "haceres" que a la vez son los productos o las materializaciones de unas capacidades (Nussbaum, 2012).

Para entender la distinción entre "capacidad" y "funcionamiento", una sociedad podría estar produciendo adecuadamente las capacidades internas para sus ciudadanos, al tiempo que, por otros canales, podría estar cortando las vías de acceso de esos individuos a la oportunidad de funcionar de acuerdo con esas capacidades. Veamos un ejemplo: una nación podría querer promover la lectura para que esta capacidad sea efectivamente incorporada por las personas. Para eso podría capacitar a profesores de primer año de educación primaria en técnicas de lectoescritura para favorecer el aprendizaje y el gusto por la lectura en los niños, pero si no potencia sus bibliotecas públicas y no disminuye el impuesto asociado a bienes como los libros y revistas para que no existan barreras monetarias a su acceso, estaría coartando el funcionamiento de aquella capacidad fortalecida.
Los Estados y las políticas públicas son centrales en la construcción de las condiciones sociales para ampliar las oportunidades de las personas. Por ello, para determinar el nivel de equidad en la dotación de una capacidad en una nación, se podría analizar el tipo de recursos que la política social ha proporcionado para ello o evaluar la incorporación de dicha capacidad o su funcionamiento en las personas que habitan esa nación.

Considerando lo anterior, un concepto que permite evaluar la dotación de una capacidad específica que tienen los habitantes de un país, como el hecho de contar con un título de educación superior, es el de trayectoria educativa. Ésta permite observar si las personas (independiente de sus características de origen como su capital cultural o nivel socioeconómico) cuentan con las mismas oportunidades para alcanzar altos niveles educativos y, con ello, realizar trayectorias educativas similares, no estando determinadas o altamente influenciadas por su condición de origen (Dávila et al., 2005).

En el nivel educativo, la distinción entre "trayectoria educativa teórica" y "trayectoria educativa real" es central. Los recorridos que debiesen realizar los sujetos en el sistema escolar, analizados en su relación con la expectativa implícita que contiene el diseño de tal sistema a través de su organización y sus determinantes se le conoce como "trayectorias escolares teóricas" (Terigi, 2009). Este tipo de trayectorias "son aquellos recorridos que siguen la progresión lineal prevista por el sistema en los tiempos marcados por una periodización estándar: que los sujetos ingresen a la escuela a la edad establecida por las normas, que transiten por los cursos escolares de manera continua, sin repetir ni abandonar la escuela, que finalicen los niveles educativos a la edad indicada para ello, habiendo realizado aprendizajes previstos en el currículum" (Terigi, 2014: 73). Estos supuestos terminan definiendo una trayectoria ideal o esperada, es decir, donde se siguen los tiempos y recorridos esperados por el sistema educativo. 
Una noción que dé cuenta de las trayectorias educativas no debiese ser normativa, sino que debe considerar las condiciones institucionales y el contexto sociocultural del estudiante que intervienen en su recorrido escolar. Entendido así, la trayectoria educativa permite enlazar la biografía de los estudiantes con las condiciones institucionales y, en lo que atañe al campo educativo, permite analizar de qué manera las condiciones institucionales del sistema educativo intervienen en la producción de estas trayectorias. Esto suele quedar oculto bajo la importancia que se asigna a las difíciles condiciones en que se desarrollan las vidas de numerosos niños, niñas y jóvenes (Donini et al., 2005; Terigi, 2014). Así se agrupan situaciones relacionadas con temas de género, de discapacidades complejas, de pertenencia a minorías étnicas específicas, de inmigrantes y de dimensión territorial. El análisis territorial en la región de América Latina y el Caribe revela que en las zonas urbanas asisten más niños y adolescentes a la educación formal, y por más tiempo, mientras que las zonas geográficas más aisladas muestran las peores tendencias en la asistencia escolar (SITEAL, s/f). La situación de la enseñanza secundaria presenta aún mayor desigualdad (Kit, 2012).

En este sentido, una trayectoria educativa "real" es diferente a la trayectoria educativa "teórica" ya que da cuenta de los verdaderos recorridos educativos realizados por los estudiantes, y que no deben verse como trayectorias fallidas, sino como expresiones de un conjunto de condiciones socioeducativas diversas que atraviesan la vida escolar. Considerar esta perspectiva implica conocer a los estudiantes y su contexto, en términos de sus condiciones estructurales, subjetivas e institucionales a las cuales se vieron enfrentados.

A partir de esta perspectiva, es posible identificar ciertos hitos transicionales que son importantes en la trayectoria educativa de un estudiante, por ejemplo, la transición de educación primaria a secundaria, de la educación secundaria general a la formación diferenciada, y la transición de la educación secundaria a la educación superior, al mercado laboral u otro. Particularmente en Chile, es en la transición a la educación superior donde existen mayores desigualdades puesto que las oportunidades para todos no son las mismas. Con la obligatoriedad de los 12 años de escolaridad, la posibilidad de no terminar la enseñanza secundaria se ha visto disminuida y la gran mayoría de la población escolar completa dicho nivel de enseñanza, pero este logro no necesariamente garantiza la continuidad de estudios superiores.

Ahora bien, a partir de una aproximación territorial, es posible suponer que el contexto educativo de los territorios pequeños presenta características particulares, que desde la etapa escolar en adelante podrían contribuir a configurar y diferenciar las trayectorias educativas.

El mayor costo asociado a la educación en territorios aislados o rurales, y la baja capacidad técnica de los niveles intermedios para gestionar financiera y pedagógicamente los centros educativos, son algunos de los problemas que enfrentan los establecimientos educacionales en territorios pequeños (Muñoz et al., 2013; Raczynski, 2012). Se suman las bajas expectativas que tienen los docentes sobre sus estudiantes; de acuerdo con algunos estudios, creen en menor proporción que sus alumnos accederán a la universidad. Ésta es una dimensión cultural relevante, dada la relación que existe entre las representaciones y expectativas docentes y sus estrategias de enseñanza (Martinic, 1999; Castillo, 2006).

Otros estudios sugieren que existen diferencias en el rendimiento académico entre zonas que concentran mayor población rural, siendo menores los resultados en este tipo de territorios. Sin embargo, al controlar por otras variables, se observa que la mayor parte de estas diferencias se explica principalmente por el nivel socioeconómico de los estudiantes de zonas rurales (Gallego et al., 2007).

En Chile y América Latina, la disponibilidad de servicios educacionales y su calidad han sido por décadas categorías condicionantes de la migración: en 
áreas rurales y ciudades con menor oferta educativa la migración ha sido la única alternativa (Raczynski, 1982). Distintas investigaciones han descrito las oportunidades educativas como factores que generan dinámicas de exclusión o expulsión del territorio, invitando a estudiantes de primaria o secundaria a abandonar su lugar de origen en tanto no satisfacen sus expectativas educativas (Rozas et al., 2009), y también han estudiado los costos académicos y subjetivos que este tipo de decisiones conllevan para ellos (Hernández et al., 2015).

Considerando el marco conceptual propuesto, el contexto chileno y el escaso nivel de conocimiento sobre el tema, surge la pregunta por las diferencias reconocibles en términos de expectativas en ES, las trayectorias educativas de jóvenes provenientes de zonas rurales hacia ese nivel, y las estrategias que planifican para continuar estudios superiores.

\section{Metodología}

Con el propósito de responder a las preguntas de investigación planteadas, el enfoque metodológico utilizado fue el de Métodos mixtos, el cual es una combinación de métodos cuantitativos y cualitativos, de enfoques y de conceptos en un sólo estudio (Tashakkori et al., 1998). Este enfoque abre la posibilidad de cotejar, contrastar y comparar diferentes tipos de datos con el mismo objetivo o foco, lo que permite validar los hallazgos que se derivan de un estudio. Tashakkori y Teddlie (1998) consideran los Métodos mixtos como óptimos para estudios complejos ya que reducen la debilidad o la limitación de cualquier método único utilizado, lo que ayuda a reducir posibles sesgos. En el contexto del trabajo propuesto, un enfoque de métodos mixtos ayuda a explorar la complejidad de la producción de expectativas y trayectorias educativas a nivel social, permitiendo así poder generar un análisis mucho más profundo.
La dimensión cuantitativa de la investigación consistió en un análisis de información primaria obtenida de las bases de datos del Sistema de Medición de la Calidad de la Educación (SIMCE), ${ }^{4}$ del Registro de Estudiantes de Chile, posteriormente denominado Sistema de Información de Estudiantes (RECHSIGE), de la Prueba de Selección Universitaria (PSU) y del Sistema de Información de Educación Superior (SIES). Por su parte, la dimensión cualitativa consistió en el análisis de transcripciones de entrevistas en profundidad y grupos focales, realizados en el marco de investigaciones recientes sobre expectativas y proyectos de vida de estudiantes de enseñanza secundaria que incluyen la dimensión territorial en el análisis.

\section{Dimensión cuantitativa}

En primer lugar, y con el propósito de analizar la evolución de las expectativas por continuar educación superior universitaria, se analizó el cuestionario aplicado por el SIMCE a estudiantes y apoderados de octavo año de primaria y de segundo año de secundaria entre los años 2004 y 2015, específicamente la pregunta por el nivel de educación más alto que les gustaría alcanzar. Para evaluar el papel de la dimensión territorial en la aspiración educativa de los adolescentes, se clasificó a los estudiantes en cuatro tipos de territorio según un criterio de escala poblacional. Primero, se identificó a estudiantes que habitaban en grandes conglomerados urbanos (compuestos por las comunas del Gran Santiago, Gran Concepción y Gran Valparaíso) y luego se agrupó a los restantes en tres categorías: estudiantes pertenecientes a territorios de 150001 a 500000 habitantes (excluyendo comunas de grandes conglomerados urbanos), la segunda de 75001 a 150000 habitantes y, por último, estudiantes pertenecientes a territorios de menos de 75000 habitantes. El tipo de análisis que se realiza de las expectativas es descriptivo; mide las brechas

\footnotetext{
${ }^{1}$ El SIMCE es el Sistema Nacional de Evaluación de Resultados de Aprendizaje y evalúa el logro de los contenidos y habilidades del currículo vigente a través de una medición que se aplica de igual manera a todos los estudiantes del país que cursan los niveles evaluados. Esta evaluación estandarizada es de carácter censal para todos los establecimientos educativos oficialmente reconocidos.
} 
entre estos grupos y da cuenta de la evolución de las expectativas de los estudiantes y sus padres entre los años 2004 y 2015.

Para analizar las trayectorias educativas considerando el entorno territorial de los estudiantes, se realizó un diseño no experimental longitudinal de tipo panel con estudiantes provenientes de distintos territorios dentro de Chile,$^{5}$ con el objetivo de hacer un seguimiento "al recorrido que sigue una cohorte de estudiantes en un tiempo determinado, a partir de su ingreso a un plan de estudios específicos" (Valle et al., 2001: 70).

Los diseños longitudinales de tipo panel sin rotación de sujetos se caracterizan por que los mismos sujetos de una cohorte son medidos repetidas veces a lo largo del tiempo, generalmente con el mismo intervalo temporal entre distintos momentos de medición y observando sus mismas variables (Arnau, 1995). De esta forma, el análisis implicó "la observación de los movimientos de una población estudiantil a lo largo de los ciclos escolares especificados en una cohorte" (García et al., 2011: 95-96). Para eso, se tomó una cohorte representativa de octavo año de primaria en 2004 y se le siguió hasta 2012 cuando, según una trayectoria teórica, lineal y exitosa (habiendo aprobado cada uno de los niveles), podría haber estado cursando su cuarto año de educación superior universitaria o haber egresado si el joven estudió una carrera técnica en un instituto profesional o en un centro de formación técnica. $\mathrm{Al}$ igual que en el ejercicio anterior, se tomó como información inicial el territorio donde el niño o niña vivía al momento de partir el trayecto observado y, según cual fuera éste, se clasificó según su comuna de domicilio en los cuatro grupos territoriales antes definidos. Bajo estos criterios, y usando un análisis descriptivo de los resultados, se observó la trayectoria educativa al final de esos nueve años para observar las diferencias entre los grupos.

Si bien, al igual que en el análisis de la evolución de las expectativas, se definieron cuatro grupos de jóvenes según territorio de origen, los resultados descritos y analizados en el marco de este artículo consideran sólo dos de ellos, los grupos más extremos (muestra de casos extremos con fines comparativos), es decir los de comunas de menos de 75000 habitantes y aquellos de grandes conglomerados. La definición de una muestra de casos extremos con fines comparativos tiene como propósito ilustrar de manera más clara el efecto del territorio tanto en las expectativas como en las trayectorias de los estudiantes por medio de las diferencias que presentan ambos grupos de jóvenes.

Finalmente, y a modo exploratorio para la cohorte de octavo año de primaria de 2004, a través de un modelo Probit se estimó el impacto en la probabilidad de ingresar a la educación superior que posee la variable territorio comparándola con otras empleadas en la regresión (rendimiento académico, sexo del estudiante, años de escolaridad de los padres, dependencia y modalidad del establecimiento donde estudia).

\footnotetext{
${ }^{5}$ La cohorte empleada corresponde a los estudiantes que cursaban octavo año de primaria en año 2004 y fueron seguidos hasta los primeros años de educación superior. Así, la cohorte puede seguir las trayectorias educacionales de los mismos estudiantes entre los años 2004 al año 2012 a través de los siguientes registros administrativos: RECH-SIGE, SIMCE, PSU y SIES. La categoría GC (Grandes Conglomerados) incluye a Santiago, Concepción y Valparaíso. Es decir: Chiguayante, Concepción, Coronel, Hualqui, Lota, Penco, San Pedro de la Paz, Santa Juana, Talcahuano, Tomé, Concón, Quilpué, Valparaíso, Villa Alemana, Viña del Mar, Cerrillos, Cerro Navia, Conchalí, El Bosque, Estación Central, Huechuraba, Independencia, La Cisterna, La Florida, La Granja, La Pintana, La Reina, Las Condes, Lo Barnechea, Lo Espejo, Lo Prado, Macul, Maipú, Ñuñoa, Pedro Aguirre Cerda, Peñalolén, Providencia, Pudahuel, Puente Alto, Quilicura, Quinta Normal, Recoleta, Renca, San Bernardo, San Joaquín, San Miguel, San Ramón, Santiago y Vitacura. Los tamaños de población para clasificar a las comunas se obtuvieron de las estimaciones de población para el año 2004 del Instituto Nacional de Estadísticas (INE) con base en el Censo de población de 2002. Para definir la comuna de residencia de los estudiantes se empleó el dato proveniente del registro de matrícula del RECH-SIGE. De este modo, las trayectorias observadas y su clasificación por tamaño de las comunas de residencia se obtuvieron a partir del registro de 2006 en octavo año de primaria. Este es un punto de partida; es posible que estos estudiantes provinieran de otras comunas o que luego se cambiaran a otras distintas, sin embargo, este es el único dato recuperable. Respecto de las trayectorias en ES cabe mencionar que éstas están comenzando y que no representan en absoluto la trayectoria final de estos jóvenes. Por lo tanto, los porcentajes se deben considerar con suficiente cuidado y recordando que son una fotografía de un momento en el tiempo y no representan en absoluto estadísticas de ingreso o egreso de la educación superior.
} 


\section{Dimensión cualitativa}

Con el propósito de tener una comprensión más subjetiva del fenómeno a estudiar, y en particular respecto a la visión de los estudiantes y sus familias respecto de la continuación de estudios superiores, se realizó un análisis de contenido de entrevistas en profundidad y grupos focales, desarrolladas en el marco de estudios recientes, que se han aproximado al fenómeno de las trayectorias educativas, la educación secundaria rural y los proyectos de vida de los estudiantes de tercero y cuarto de secundaria en Chile (Atria et al., 2016; UDP, 2016b; Zamorano et al., 2017). Específicamente, estos estudios indagaron en las percepciones de estudiantes de sectores rurales de $2^{\circ}$ y $4^{\circ}$ año de secundaria y de sus padres y apoderados, respecto de los proyectos de vida de los jóvenes, de sus expectativas educativas-laborales, de las capacidades, herramientas y estrategias para concretar dichos proyectos, y a los desafíos que estos implicaban. Todo lo anterior pudo ser abordado a través de preguntas como:

- ¿Qué te/les gustaría hacer cuando terminen la secundaria?

- ¿Tienen planes de seguir estudios superiores?

- Ese interés que tienes/n, ¿cómo se originó?
- ¿Qué crees/n que necesitas/n hacer para lograr lo que quieres $/ \mathrm{n}$ hacer a futuro?

- ¿Qué herramientas te/les ha entregado el colegio que te/les sirve/n para lograr tus/sus objetivos o proyectos?

El análisis de contenido utilizado es una técnica de interpretación de un texto escrito, grabado o filmado (Abela, 2002), que consiste en un análisis de la comunicación que, a través de la codificación y el conteo sistemático, permite inferencias respecto a la condición de producción/recepción de los datos (Bardin, 2002). Este análisis incluye la definición de códigos antes de buscarlos en los datos y, de esta manera, es una metodología deductiva, considerada objetiva porque es explícita sobre cómo se codificará el contenido (Rice et al., 1999).

Estos estudios tenían como unidades de análisis a estudiantes, padres y establecimientos educativos de enseñanza secundaria, en que la dimensión territorial se operacionalizaba en la distinción rural/urbana. En especial este tipo de análisis fue significativo para identificar estrategias para la continuación de estudios.

A modo de resumen, se presentan en la tabla 2 las distintas fuentes de información utilizadas en la investigación y a qué ámbito del análisis corresponden.

Tabla 2 . Fuentes de información secundarias utilizadas en el estudio

\begin{tabular}{|l|l|l|}
\hline \multicolumn{1}{|c|}{ Fuente } & \multicolumn{1}{|c|}{ Información contenida } & \multicolumn{1}{c|}{ Permite análisis de } \\
\hline BBDD-SIMCE & $\begin{array}{l}\text { Pregunta a estudiantes y padres sobre el nivel de educación } \\
\text { más alto que les gustaría alcanzar (2004 y 2015). }\end{array}$ & $\begin{array}{l}\text { Evolución de expectativas de educación universitaria en } \\
\text { estudiantes y padres, y sus brechas. }\end{array}$ \\
\hline BBDD-RIECH, PSU y SIES & $\begin{array}{l}\text { Seguimiento de cohorte de estudiantes según tipo de } \\
\text { territorio de origen entre 2004, cuando estaban en octavo } \\
\text { año de primaria, hasta 2012. }\end{array}$ & $\begin{array}{l}\text { Análisis de trayectorias de estudiantes entre educación } \\
\text { escolar y educación superior. Modelo Probit. }\end{array}$ \\
\hline $\begin{array}{l}\text { Entrevistas en profundidad } \\
\text { y grupos focales de } \\
\text { estudios sobre trayectorias } \\
\text { educativas }\end{array}$ & $\begin{array}{l}\text { Transcripciones de información cualitativa levantada } \\
\text { (entrevistas en profundidad y grupos focales con } \\
\text { estudiantes de tercero y cuarto de secundaria y sus padres). }\end{array}$ & $\begin{array}{l}\text { Análisis denso y subjetivo de expectativas, trayectorias y } \\
\text { estrategias para lograr estudios universitarios de estudiantes }\end{array}$ \\
\hline
\end{tabular}

Fuente: elaboración propia. 


\section{Principales resultados}

\section{Expectativas en continuación de estudios universitarios: diferencias por territorios y su evolución en la última década}

El interés por contar con educación superior, en particular universitaria, va en aumento. Pero ¿cómo ha evolucionado esto a nivel de las expectativas de estudiantes y familias de zonas rurales y urbanas en la última década? En la tabla 3 se puede observar cómo se distribuye y evoluciona, entre 2009 y 2015 , la aspiración de terminar una carrera universitaria en los estudiantes de los dos tipos de territorio considerados.

La expectativa de completar una carrera en la universidad ha ido ganando fuerza en los últimos años entre los estudiantes de territorios más pequeños o rurales. Si en 2009 el 56\% de este grupo declaraba su interés por completar una carrera universitaria, en 2015 la cifra alcanzó un 65\%. Con este incremento, y considerando el menor aumento de la expectativa de jóvenes de centros urbanos, la brecha entre ambos grupos de estudiantes es cada vez menor. Este mismo fenómeno se observa en los análisis cualitativos, donde los propios estudiantes de sectores rurales mencionan que ahora la mayoría de los estudiantes están interesados en continuar estudios superiores; y que además son los propios docentes y directivos de los colegios los que hoy los incentivan a seguir ese camino.

Sí, la verdad es que todos piensan en estudiar. Son muy pocos los que tienen en mente "no, no voy a estudiar ahora", son muy pocos y casi todos están proyectados a futuro para sacar su profesión (estudiante cuarto año de secundaria, Liceo Rural).

De segundo medio, primero medio, ya nos están diciendo que tenemos que ir a prepararnos para ver nuestras carreras, que nos vamos por el lado de lo que queremos estudiar, que lo pensemos, que tenemos que ya de primero estarle poniendo bueno para la PSU (estudiante cuarto año de secundaria, Liceo Rural).

Tabla 3. Porcentaje de estudiantes de $8^{\circ}$ básico y $2^{\circ}$ medio, de cada grupo territorial, a quienes les gustaría terminar una carrera en la universidad

\begin{tabular}{|c|c|c|c|c|c|}
\hline \multirow{2}{*}{ Año } & \multicolumn{2}{|c|}{$\begin{array}{l}\text { Estudiantes de grandes } \\
\text { conglomerados urbanos }\end{array}$} & \multicolumn{2}{|c|}{$\begin{array}{c}\text { Estudiantes de territorios con } \\
\text { menos de } 75.000 \text { habitantes }\end{array}$} & \multirow{2}{*}{$\begin{array}{l}\text { Brecha (puntos } \\
\text { porcentuales)* }\end{array}$} \\
\hline & $\%$ & $N$ & $\%$ & $N$ & \\
\hline 2009 & $67 \%$ & 54416 & $56 \%$ & 31010 & -11 \\
\hline 2010 & $69 \%$ & 67334 & $56 \%$ & 35312 & -13 \\
\hline 2011 & $69 \%$ & 54329 & $61 \%$ & 37211 & -8 \\
\hline 2013 & $70 \%$ & 95907 & $61 \%$ & 60581 & -9 \\
\hline 2014 & $70 \%$ & 102737 & $65 \%$ & 66532 & -5 \\
\hline 2015 & $71 \%$ & 107903 & $65 \%$ & 71744 & -6 \\
\hline
\end{tabular}

Fuente: elaboración propia

*Diferencia estadísticamente significativa al $5 \%$ en todos los años analizados. 
Como se observa en la tabla 4, el aumento de las aspiraciones a continuar estudios también se refleja en la opinión de padres y apoderados. Se observa que entre 2004 y 2015, la expectativa de los padres que habitan territorios pequeños de que sus hijos logren una educación universitaria se ha duplicado, pasando de un 34\% en 2004 a un 67\% en 2015, con lo que, al igual que en el caso de los jóvenes, disminuye la brecha de expectativas en relación con grandes conglomerados urbanos (de 19 a 6 puntos porcentuales). Este aumento de las expectativas de las familias de los estudiantes para que prosigan una educación superior también se observa en el relato de los apoderados de los estudiantes de sectores rurales. Relato que se fundamenta en la precariedad y dificultad que significa vivir y trabajar en el sector rural, frente al cual la idea de ser profesional y salir del campo es el mejor camino que pueden tomar.

Sácate una carrera hija, le digo yo, y estudia. No es fácil la pega del campo. Si tienen que estudiar, le digo yo, esfuérzate, sé que puedes, porque a usted aquí gracias a Dios le ha ido bien. Es como que se le abrió la mente donde ve estudiar a su otra hermana (apoderado estudiante cuarto año de secundaria, Liceo Rural).
Ellos tienen la expectativa de irse a estudiar, tener otra vida fuera de acá de donde vivimos (Apoderado Estudiante cuarto año de secundaria, Liceo Rural).

Se observa que el acceso a la educación superior como un elemento del proyecto de vida imaginado por los adolescentes y sus familias aparece de manera transversal, y que ha comenzado a perder el anclaje territorial que antes lo caracterizaba. Este dato es especialmente relevante para pensar en una sociedad con menos desigualdades ya que, según los datos Casen de 2015, sobre ingresos económicos y empleabilidad en Chile, la brecha salarial entre tener ES y no tenerla es importante.

En un escenario en el que disminuyó la brecha en las aspiraciones de continuidad de estudios universitarios entre los estudiantes y las familias de zonas rurales respecto de las urbanas, surge la interrogante acerca de si esa expectativa se condice con la realidad. Es decir, si las trayectorias educativas hacia la educación superior de jóvenes de ambos tipos de territorios se comportan relativamente similares. Esa tendencia daría cuenta de la equidad o inequidad de oportunidades en estos distintos tipos de territorios.

Tabla 4. Porcentaje de apoderados de $8^{\circ}$ básico y $2^{\circ}$ medio, por grupo territorial, a quienes les gustaría que sus hijos terminaran una carrera en la universidad

\begin{tabular}{|c|c|c|c|c|c|}
\hline \multirow{2}{*}{ Año } & \multicolumn{2}{|c|}{$\begin{array}{c}\text { Apoderados de grandes } \\
\text { conglomerados urbanos }\end{array}$} & \multicolumn{2}{c|}{$\begin{array}{c}\text { Apoderados de territorios con } \\
\text { menos de 75 000 habitantes }\end{array}$} & \multirow{2}{*}{$\begin{array}{c}\text { Brecha (puntos } \\
\text { porcentuales)* }\end{array}$} \\
\cline { 2 - 5 } & $\%$ & $\boldsymbol{N}$ & $\%$ & $\boldsymbol{N}$ & -19 \\
\hline 2004 & $53 \%$ & 57509 & $34 \%$ & 26446 & -18 \\
\hline 2007 & $55 \%$ & 51446 & $37 \%$ & 26054 & -15 \\
\hline 2009 & $66 \%$ & 48758 & $51 \%$ & 25569 & -13 \\
\hline 2010 & $69 \%$ & 60988 & $56 \%$ & 30544 & -12 \\
\hline 2011 & $64 \%$ & 47056 & $52 \%$ & 30736 & -10 \\
\hline 2013 & $69 \%$ & 81657 & $59 \%$ & 53856 & -6 \\
\hline 2014 & $71 \%$ & 83833 & $65 \%$ & 57522 & -6 \\
\hline 2015 & $73 \%$ & 89476 & $67 \%$ & 64540 & \multicolumn{2}{|c|}{} \\
\hline
\end{tabular}

Fuente: elaboración propia a partir de datos de la Agencia de la Calidad, Encuesta SIMCE, 2004 a 2015.

*Diferencia estadísticamente significativa al $5 \%$ en todos los años analizados. 


\section{Trayectorias educativas hacia la educación superior: análisis según tipo de territorio de origen de los estudiantes}

Para responder esta interrogante se realizó un estudio de trayectorias de estudiantes provenientes de territorios de distinta escala. Para ello se seleccionó y levantó información secundaria sobre una cohorte representativa de estudiantes que cursaban octavo año de primaria en 2004 y se le siguió hasta 2012, caracterizando su paso por los distintos niveles educativos a lo largo de esos ocho años. Si bien este corpus de datos no captura las más recientes tendencias sobre acceso a educación superior, permite profundizar sobre las diferencias de oportunidades que entregan distintos contextos territoriales en un momento de tiempo. En la figura 1 se presenta la distribución de dichas trayectorias.

Como se observa en la figura 1, en el egreso de educación secundaria no existen diferencias relevantes en función del tipo de territorio que se habita, pues cerca del 90\% de los jóvenes, independientemente del territorio, culmina la etapa de educación escolar. En la enseñanza secundaria hay una mayor preferencia por la educación técnico-profesional entre los estudiantes de territorios pequeños, dado que un $46 \%$ de ellos opta por esta modalidad de enseñanza, en comparación con el 39\% de estudiantes de grandes conglomerados urbanos.

En el tránsito de la educación escolar a la educación superior, en cambio, se presentan desigualdades en función del territorio. Entre estudiantes que habitan grandes conglomerados urbanos es mayor la proporción de quienes acceden a este nivel educativo: un 70\%, mientras que sólo el 55\% de los estudiantes que viven en territorios pequeños lo hace. Si bien en ambos tipos de territorios la preferencia más marcada entre los jóvenes es la educación superior universitaria, es mayor la proporción de estudiantes de grandes conglomerados los que acceden a este tipo de instituciones, en comparación con estudiantes de territorios pequeños o rurales.

\section{Figura 1. Trayectoria de la cohorte (\% sobre el total de cada grupo territorial)}

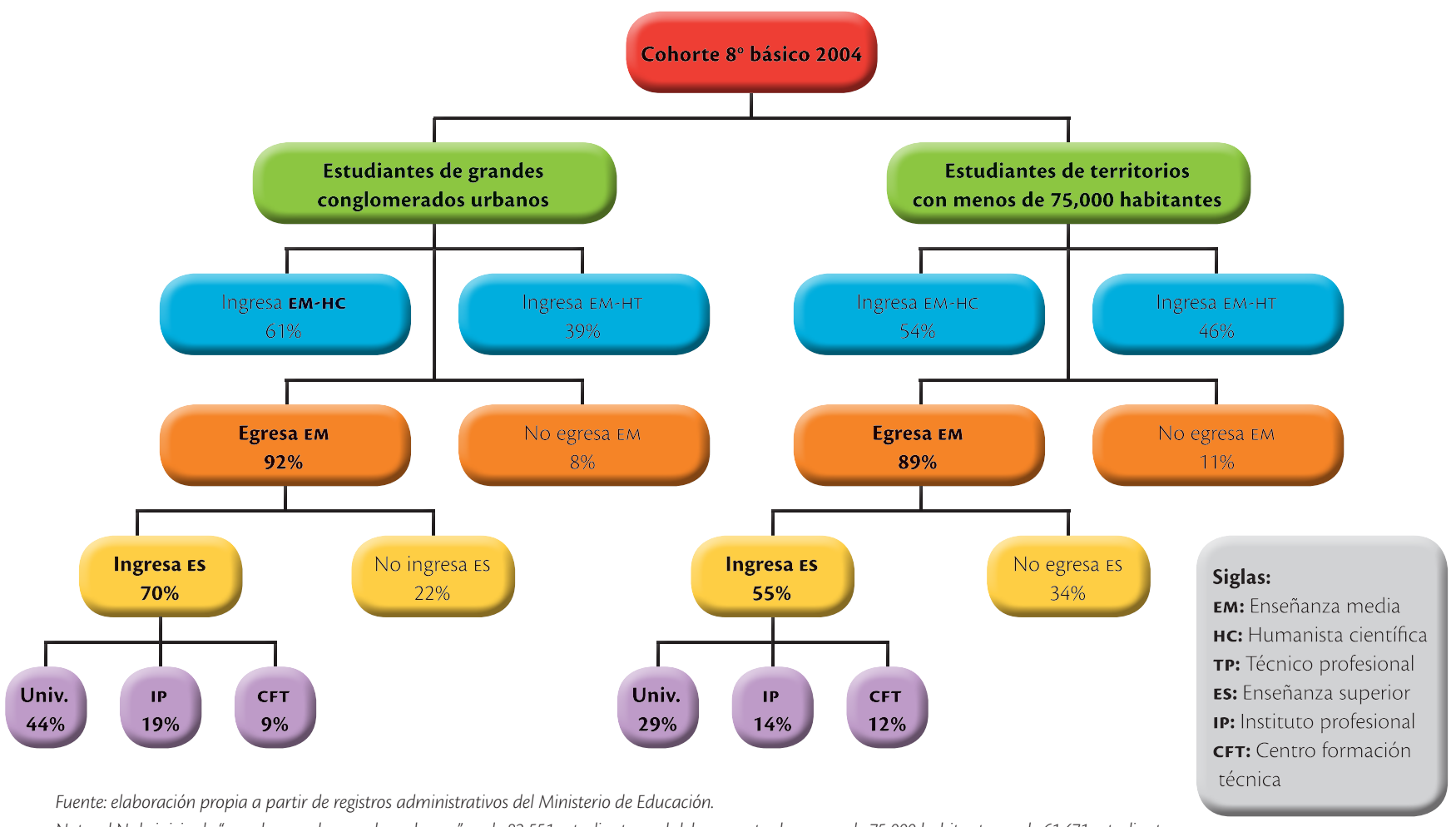

Nota: el N de inicio de "grandes conglomerados urbanos" es de 82551 estudiantes y el del segmento de menos de 75000 habitantes es de 61.471 estudiantes. 
$\mathrm{Al}$ indagar qué otras variables pueden estar incidiendo en estas trayectorias, se aprecia que al controlar por rendimiento académico (puntaje promedio SIMCE), el territorio sigue siendo un componente explicativo en la configuración de estas trayectorias. Es así como estudiantes de territorios de menor escala con el mismo rendimiento académico en SIMCE tienen menos probabilidades de ingresar a la educación superior que estudiantes de grandes conglomerados urbanos.

En un contexto en el que estudiantes de territorios pequeños tienen menor probabilidad de continuar hacia la educación superior en comparación con sus pares de grandes conglomerados urbanos, cabe preguntarse cómo influyen estas diferencias de oportunidades en los proyectos de vida de los primeros.

\section{La migración como estrategia clave para la continuación de estudios superiores}

Como se ha visto, la aspiración de continuar estudiando una vez finalizada la enseñanza secundaria es parte de los proyectos de vida de los jóvenes chilenos actualmente, y se ve una menor influencia del territorio en donde se habita en la construcción de esa aspiración. Sin embargo, el análisis de trayectorias confirma que el territorio sí es un factor condicionante para la continuidad de estudios.

Esto lleva a la pregunta: ¿cómo es la relación de los jóvenes con el territorio cuando el anhelo de seguir estudiando se ve limitado por éste? Los análisis cualitativos realizados dan cuenta de una cierta tensión en el vínculo de los jóvenes con el territorio en esta etapa de su vida. Territorios pequeños, aislados o rurales, son vistos por los adolescentes como espacios carentes de oportunidades o con posibilidades muy restringidas para seguir estudiando o acceder a un trabajo. Es en esta tensión donde surge la idea de migrar.

Las principales razones de quienes optan por migrar y así desplazarse hacia centros urbanos se relacionan con poder darle continuidad a la educación secundaria en una modalidad de enseñanza que no se encuentra disponible en el territorio (humanistacientífica o técnica-profesional) o el interés de continuar con la educación superior en el ámbito técnico o profesional. En otros casos, se observa que la necesidad de migrar proviene de la búsqueda de alternativas laborales que no encuentran en su territorio, o de la posibilidad de formarse en instituciones de policía o de las fuerzas armadas. Esta argumentación queda reflejada en la siguiente cita:

Yo creo que, por seguir el sueño de uno, si fuera por mí, por lo que quiero estudiar, tendría que irme a Santiago a estudiar (estudiante cuarto año de secundaria, Liceo Rural).

Así mismo se constata, entre los jóvenes de estos territorios, que la migración se encuentra interiorizada como posibilidad desde temprana edad, ya que se visualiza no sólo como un paso necesario para concretar las propias aspiraciones sino también - como emerge en las entrevistas - se ve como un hito relevante del desarrollo personal, connotando socialmente el grado de madurez y autonomía que se tiene. Así mismo, los estudiantes tienen plena conciencia de que tomar esta opción implica un sacrificio subjetivo alto al tener que dejar sus familias, pero están dispuestos a realizarlo.

Yo creo que igual sería como una forma de madurar también salir, si es que tuviéramos que estudiar afuera, sería como una forma de madurar más rápido, igual nos alejaríamos de los papás, entonces, igual ahí ya cambia algo (estudiante cuarto año de secundaria, Liceo Rural).

Si me voy a estudiar a Santiago no estaría con el apoyo así al lado de mi mamá, me faltaría como mi mamá al lado. Quizás eso. Estaría en la casa de alguien más, no con ella, igual es como distinto. No es lo mismo que el apoyo familiar (estudiante de tercer año de secundaria, Liceo Rural). 
El acto de emigrar es vivido también como un sacrificio significativo en las familias de los estudiantes, en tanto el alejamiento del territorio implica asumir un costo económico importante que es necesario realizar y, en términos afectivos, implica un prolongado alejamiento de los seres queridos. Esta tensión se observa en el discurso de los apoderados de los estudiantes de sectores rurales, ya que los costos de un hijo estudiando implica sacrificar otros gastos familiares igual de relevantes, o incluso optar por que sólo uno de sus hijos pueda proseguir estudios superiores. A pesar de ello perciben que es necesario hacerlo para generar mejores condiciones de vida para sus hijos.

Mi hija tiene como primera prioridad Geología, una carrera que dura siete años, en la sede de Concepción. Una carrera carísima, con un instrumental que no lo estudia cualquiera, pero resulta que para irse a estudiar tendría que irse de la comuna. Nosotros podríamos financiar el estudio de ella, pero tendría que sacrificar a mi hijo menor que es el que viene justo después. Entonces tampoco sería justo porque uno quiere que se eduquen todos y parejos (apoderada estudiante cuarto año de secundaria. Liceo Rural).

Aparte a nosotros igual nos aumenta el costo porque nosotros tenemos que tomarlos y trasladarnos, tener un lugar donde estén allá, tener en cuenta los viajes para que el fin de semana vengan nuevamente, la preocupación de que estén allá solos [...]. Yo le dije a mi hija: "si tienes que irte a la China, a la China te apoyo y voy a rescatarte donde sea, aunque a lo mejor me falte a mí para comer, veremos de dónde sacamos la plata (apoderada estudiante cuarto año de secundaria. Liceo Rural).

Si bien el territorio rural es vivido como una amenaza para la materialización de los proyectos de vida de muchos jóvenes, se observan disposiciones diversas respecto a su vínculo con él. Para algunos representa un lugar querido, en el que permanecen vínculos afectivos importantes y al que esperan retornar más adelante. Para otros, no se observa un apego importante, por lo que la permanencia en otra localidad podría transformarse en algo más permanente. Así mismo, es sabido que migrar a otro territorio no siempre está dentro del margen de posibilidades de todas las familias de zonas rurales. Es común que aquellos jóvenes que no manifiestan un proyecto de vida claro o no cuentan con las oportunidades para concretarlo permanezcan en sus territorios de origen y tempranamente se vinculen al mundo del trabajo reproduciendo así la cultura local.

Si bien es cierto que los discursos de los jóvenes de zonas rurales y sus familias son diversos al indagar en sus expectativas y posibilidades de concreción, en el discurso siempre permanece la imagen de una falta, de un aspecto que coarta sus libertades por el sólo hecho de encontrarse lejos de las oportunidades.

\section{Discusión}

Analizar el vínculo entre educación y territorio es relevante en tanto la educación es una de las vías esenciales para el desarrollo de las capacidades que habilitan a las personas para decidir sobre sus vidas y poner en acción las estrategias necesarias para alcanzar sus metas y aspiraciones.

Lo primero que se puede observar, y que confirma lo ya mencionado en el marco teórico, es que en Chile, debido a la expansión y obligatoriedad de la educación secundaria, no se perciben brechas entre los jóvenes de los distintos territorios respecto al egreso de dicho nivel educativo. En otras palabras, no habría diferencias significativas entre las trayectorias educativas "teóricas" y aquellas "reales" al comparar a los jóvenes que estudian en estos distintos contextos territoriales en las últimas décadas.

Junto a ello, se percibe un aumento significativo de las aspiraciones vinculadas con la continuidad de estudios. Más allá de dónde se viva, se observa una disminución de la brecha de expectativas de continuar estudiando en la educación superior, tanto en 
adolescentes como en padres de distintos contextos territoriales. Fenómeno que estaría dando cuenta de un desanclaje territorial en la dimensión educacional de los proyectos de vida y de que las "preferencias adaptativas" no estarían operando en las decisiones vocacionales de la población rural, como podía quizás verse décadas atrás.

Este fenómeno creciente de aumento de expectativas en estas materias y de disminución de la brecha según el territorio donde se habite, estaría dando cuenta de un desarrollo importante a nivel de las "capacidades", y que estaría dotando efectivamente a las personas de "libertades reales" para que puedan realizar aquello que efectivamente desean, con lo que éstos se encontrarían - en términos de sus expectativas - cada vez menos determinados por el medio en el que habitan. Desde el enfoque de capacidades, y mirando las expectativas de estudiantes y de apoderados, se estarían ampliando las oportunidades para decidir lo que se quiere hacer y ser.

A pesar de lo anterior, en este escenario en el que la influencia territorial sobre las aspiraciones va disminuyendo, aún se observan desigualdades territoriales a la hora de materializar dichas aspiraciones, es decir, a la hora de pasar a los "funcionamientos" de las capacidades. De alguna forma, alcanzar estos anhelos sigue estando condicionado por el territorio que se habita: jóvenes y adolescentes de grandes territorios ingresan en mayor proporción que los de territorios pequeños a la educación superior universitaria.

Por tanto, aún existirían condiciones desiguales en el entorno que estarían afectando el despliegue de esta capacidad, con lo que se generarían diferencias importantes en términos de las trayectorias educativas reales postsecundarias. Lo anterior se torna más complejo cuando hay evidencia de que, aún con igual nivel de resultados de aprendizaje, estudiantes de territorios de menor escala tienen menos probabilidades de ingresar a la educación superior que estudiantes de grandes conglomerados urbanos.
$\mathrm{Al}$ considerar estas condiciones adversas, los jóvenes de sectores rurales elaboran proyectos de vida en los que la migración aparece como una estrategia clave para la continuidad de estudios en educación superior. Migración que puede tener como correlato la concentración de mejores condiciones y mayores capacidades en los grandes conglomerados urbanos y la continua depreciación de las zonas más pequeñas o aisladas.

Este dilema explicita de manera extrema una clásica tensión del desarrollo humano: potenciar las capacidades y agencia de las personas versus potenciar el desarrollo de los territorios. El enfoque de las capacidades ha definido como propósito final del desarrollo, el fortalecimiento y la libertad de las personas, las que deben ser potenciadas a través de la creación de recursos en los entornos que hagan posible dicho fortalecimiento. Potenciar a las personas requiere potenciar los entornos con los que estas interactúan, de forma tal que el desarrollo aparecería como un todo imbricado. Es esta la perspectiva con la cual debe pensarse el diseño de políticas públicas que fomenten el desarrollo a nivel personal y territorial.

En este sentido, distintas iniciativas públicas podrían contribuir en la reducción de las desigualdades territoriales observadas en esta investigación. Una de ellas es la recientemente aprobada ley de Nueva Educación Pública, que busca aportar a la descentralización y autonomía de los niveles intermedios en la gestión educativa, y fomentar la participación de la comunidad local y la colaboración entre escuelas para mejorar la capacidad de gestión y la educación pública en distintos territorios. Es probable que la actual política de gratuidad en educación superior podrá aportar a ello también, así como la generación de nuevas universidades y centros de formación técnica estatales en todas las regiones del país.

Si bien estas iniciativas representan un avance en la línea de hacer más equitativas las oportunidades educativas independientemente del territorio, el escenario creciente de las aspiraciones educativas entre los jóvenes y el enfoque de las capacidades impone el 
desafio de pensar en mecanismos que permitan entregar un apoyo mucho más focalizado y pertinente al contexto. Se requiere favorecer políticas educativas que permitan efectivamente que los jóvenes de zonas más excluidas o aisladas logren acceder y terminar

\section{Referencias}

Abela, Jaime (2001), Las técnicas de análisis de contenido: una revisión actualizada, Sevilla, Centro de Estudios Andaluces <https://www.centrodeestudiosandaluces. es/publicaciones/tecnicas-de-analisis-de-contenidouna-revision-actualizada> [Consulta: junio de 2020].

Arnau, Jaume (1995), Diseños longitudinales aplicados a las ciencias sociales y del comportamiento, México, Editorial Limusa.

Atria, Raúl, Camilo Araneda, Rodrigo Fernández, Loreto de la Fuente, Ismael Puga y Pablo Soto (2016), Proyectos de vida y oportunidades en la educación media. Nuevas demandas sociales al sistema escolar chileno, Santiago de Chile, Ministerio de Educación, <https://centroestudios. mineduc.cl/wp-content/uploads/sites/100/2017/07/ INFORME-FINAL-F911453.pdf> [Consulta: mayo de 2019].

Bardin, Laurence (2002), Análisis de contenido, Madrid, Ediciones AKAL.

Brunner, José Joaquín (2015), “Medio siglo de transformaciones de la educación superior chilena: un estado del arte", en A. Bernasconi (ed.), La educación superior en Chile: transformación, desarrollo y crisis, Santiago de Chile, Ediciones Universidad Católica de Chile, pp. 21-107.

Castillo, Jorge (2013) "Movilidad social y educación en Chile", en J. F. Tezanos (ed.), Juventud, cultura y educación. Perspectivas comparadas en España y Chile, Madrid, Siglo XXI, pp. 267-286.

Castillo, Jorge (2006), “¿Representación institucional del 'rol docente' o representación del 'joven popular' como carreras técnicas y/o profesionales. Estrategias que logren mejorar la calidad educativa de esas zonas y que ofrezcan oportunidades compatibles con los vínculos afectivos y territoriales que estos jóvenes han desarrollado con su lugar de origen.

alumno? Algunas reflexiones respecto a la tensión sobre la que se fundamenta 'el proceso educativo' en la enseñanza secundaria en contextos de pobreza", Revista Última Década, núm. 24, pp. 11-36.

Consejo Nacional de Educación (CNED) (2016), Tendencia Índices 2016, Santiago de Chile, GNED, <https:// www.cned.cl/sites/default/files/tendencias_indices_ junio_2016.pdf> [Consulta: junio de 2019].

Concha, Claudia (2013), "Trayectorias sociales de sujetos rurales que por primera generación acceden a la educación superior universitaria en la Región del Maule, Chile”, Sociedad Hoy, vol. 24, pp. 55-68.

Dávila, Oscar, Felipe Ghiardo y Carlos Medrano (2005), Los desheredados: trayectorias de vida y nuevas condiciones juveniles, Santiago de Chile, Editorial CIDPA.

Donini, A., J. Gorostiaga y M. Pini (2005), Informe hemisférico comparativo de políticas y estrategias para la prevención del fracaso escolar, Buenos Aires, Universidad Nacional de San Martín.

Gallego, Francisco, Carlos Rodríguez y Enzo Sauma (2007), Provisión de educación en zonas rurales de Chile: incentivos, costos y calidad. Camino al Bicentenario, propuestas para Chile, Santiago de Chile, Universidad Católica de Chile.

García, Octaviano y Concepción Barrón (2011), "Un estudio sobre trayectoria escolar de los estudiantes de doctorado en Pedagogía”, Perfiles Educativos, vol. 33, núm.131, pp. 94-113, <https://www.redalyc.org/ pdf/132/13218531007.pdf> [Consulta: mayo de 2019]. 
Hernández, Macarena y Dagmar Raczynski (2015), "Elección de escuela en Chile. De las dinámicas de distinción y exclusión a la segregación socioeconómica del sistema escolar", Estudios pedagógicos, vol. 41, núm. 2, pp. 127-141, DOI: https://dx.doi.org/10.4067/S071807052015000200008 [Consulta: marzo de 2019].

Instituto Nacional de la Juventud (INJUV) (2017), Octava Encuesta Nacional de Fuventud, Santiago de Chile, Instituto Nacional de Juventud, <http://www.injuv.gob.cl/ storage/docs/Libro_Octava_Encuesta_Nacional_de_ Juventud.pdf $>$ [Consulta: mayo de 2019].

Kit, Irene (coord.) (2012), Completar la escuela. Un derecho para crecer, un deber para compartir, Panamá, UNICEF.

Martinic, Sergio (1999), "Las representaciones de la desigualdad y la cultura escolar en Chile", Proposiciones, vol. 34, pp. 1-10.

Ministerio de Desarrollo Social (MDS) (2015a), Informe de Desarrollo Social 2015, Santiago de Chile, MDS, <http:/ / www.ministeriodesarrollosocial.gob.cl/pdf/upload/ IDS2.pdf> [Consulta: febrero de 2019].

MDS (2015b), CASEN 2015. Educación. Síntesis de resultados, Santiago de Chile, Ministerio de Desarrollo Social, <http://observatorio.ministeriodesarrollosocial.gob.cl/ casen-multidimensional/casen/docs/CASEN_2015_ Resultados_educacion.pdf> [Consulta: abril de 2019].

Muñoz, César y Gonzalo Muñoz (2013), Desigualdad territorial en el sistema escolar: la urgencia de una reforma estructural a la educación pública en Chile, Santiago de Chile, Programa Cohesión Territorial para el Desarrollo, Centro Latinoamericano para el Desarrollo Rural.

Nussbaum, Marta (2012), Crear capacidades. Propuesta para el desarrollo humano, Barcelona, Paidós.

Programa de Naciones Unidas para el Desarrollo (PNUD) (2012), Informe de Desarrollo Humano en Chile: Bienestar subjetivo en Chile: el desafio de repensar el desarrollo, Santiago de Chile, PNUD.

PNUD (2017), Desiguales: orígenes, cambios y desafios de la brecha social en Chile, Santiago de Chile, PNUD.

PNUD-UNICEF (2014), El papel de la educación en la formación del bienestar subjetivo para el desarrollo humano: una revisión al caso chileno, Santiago de Chile, PNUD-UNICEF
Puga, Ismael, Raúl Atria, Rodrigo Fernández y Camilo Araneda (2017), "Proyectos de vida y oportunidades en la educación media. Nuevas demandas sociales al sistema escolar chileno", Última década, núm. 47, pp. 118-153.

Raczynski, Dagmar (2012), "Realidad de la educación municipal en Chile: ¿liderazgo del sostenedor municipal?”, en J. Weinstein y G. Muñoz (eds.), ¿Qué sabemos sobre los directores de escuela en Chile?, Santiago de Chile, Fundación Chile-CEPPE, pp. 181-218.

Raczynski, Dagmar (1982), "Origen, destino y composición sociodemográfica de la migración interna", Notas Técnicas, núm. 50, Santiago de Chile, CIEPLAN.

Rice, Pranee Liamputtong y Douglas Ezzy (1999), "Qualitative research methods: a health focus", International Journal of Epidemiology, vol. 30, núm. 1, pp. 185.

Rozas, Carolina y Claudia Lara (2009), "Jóvenes rurales y educación media: un estudio exploratorio acerca de la relación entre jóvenes rurales escolarizados de la Provincia de Cachapoal, Región de O’Higgins y la educación media en Chile", en P. Castro (ed.), Fuventud $y$ enseñanza media en Chile del Bicentenario: antecedentes de la revolución pingüina, Santiago de Chile, Observatorio Chileno de Políticas Educativas, OPECH, pp. 128-163. Sen, Amartya (1999), Desarrollo y libertad, Barcelona, Editorial Planeta.

SITEAL (s/f), "La asistencia escolar en la actualidad. Trayectorias educativas en ocho países de América Latina", en Atlas de las desigualdades educativas en América Latina, cap. 3. Buenos Aires, IIPE-UNESCO/OEI, <http://Atlas.siteal.org/capitulo_3> [Consulta: mayo de 2020].

Tashakkori, Abbas y Charles Teddlie (1998), "Mixed methodology: combining qualitative and quantitative approaches", Applied Social Research Series, vol. 46, Londres, Thousand Oaks..

Terigi, F. (2014), "Trayectorias escolares e inclusión educativa: del enfoque individual al desafio para las políticas educativas", en A. Marchesi, R. Blanco, y L. Hernández, Avances y desafios de la educación inclusiva en Iberoamérica, Madrid, Organización de Estados Iberoamericanos. 
Terigi, F. (2009), Las trayectorias escolares. Del problema individual al desafio de política educativa, Buenos Aires, Ministerio de Educación.

Universidad Diego Portales (UDP) (2016a), Estudio niños, niñas, adolescentes y jóvenes fuera de la escuela. Caracterización y análisis de la demanda para una modalidad de Escuelas de Segunda Oportunidad, Santiago de Chile, Centro de Políticas Comparadas de Educación, UDP.

UDP (2016b), Estudio sobre transiciones educativas en la enseñanza media y definiciones de la política para este nivel educativo, Santiago de Chile, Centro de Políticas
Comparadas de Educación, UDP.

Valle, Rosamaría, Graciela Rojas y Ariadna Villa (2001), "El análisis de las trayectorias escolares en la UNAM: un método de análisis", en Deserción, rezago y eficiencia terminal en la IES, propuesta metodológica para su estudio, México, Asociación Nacional de Universidades e Instituciones de Educación Superior (ANUIES), pp. 51-74.

Zamorano, Humberto, Víctor Serrano y Hernaldo Zamora (2017), Estudio de establecimiento de educación media en territorios rurales, Santiago de Chile, Grupo de Estudios Económicos y Territoriales PULSO. 